\title{
The kissing puncta: an under-reported and stubborn cause of epiphora
}

\author{
Varajini Joganathan ${ }^{1} \cdot$ Bhupendra C. K. Patel ${ }^{2} \cdot$ Raman Malhotra $^{3} \cdot$ Jonathan H. Norris $^{1}$
}

Received: 12 June 2018 / Accepted: 27 August 2018 / Published online: 29 November 2018

(c) The Royal College of Ophthalmologists 2018

\begin{abstract}
Background 'Kissing puncta' (KP) or punctal apposition is an anatomical phenomenon sparsely reported in the English literature. We describe our experience of managing chronic epiphora in patients with punctal apposition.

Methods A retrospective audit of five patients (nine eyes) with KP associated with epiphora. Data including: presenting symptoms, physical signs and surgical outcomes were collected.

Results Five patients aged between 66 and 77 years were reviewed. Common clinical features were: chronic epiphora, involutional eyelid laxity, kissing puncta (present at all phases of the blink) and reduced upper and lower margin-reflex distances. Medial upper eyelid ptosis with orbital fat prolapse was a prominent feature.

Four patients (nine eyes underwent eyelid-tightening surgery to restore normal anatomical position of the puncta. Only one of the four patients achieved improvement in epiphora at 3 months. One patient with continued epiphora underwent subsequent dacrocystorhinostomy with improvement in symptoms. The fifth patient had mild laxity and underwent dacrocystorhinostomy at first instance, with no improvement in symptoms, despite surgical success.

Conclusions The KP sign is commonly found in those with involutional eyelid changes. Epiphora is present in variable degrees in the presence of punctal apposition. Restoration of normal punctal position with eyelid-tightening surgery does not always confer an improvement in epiphora. Surgical management in the setting of KP is therefore challenging with a guarded prognosis. Symptomatic patients with KP should be counselled accordingly.
\end{abstract}

\section{Introduction}

Epiphora may occur due to anatomical malposition of the puncta, or even misalignment whereby the superior and inferior puncta appose and occlude each other leading to mechanical obstruction of tear drainage [1]. The anatomical phenomenon of punctal apposition was first described as 'kissing puncta' (KP) by Bartley et al. in 1993 [1]. This

This case series was presented at the British Oculoplastic Society Meeting, London, June 2017.

Jonathan H. Norris

jonathan.norris@ouh.nhs.uk

Oxford Eye Hospital, John Radcliffe Hospital, Oxford, UK

2 Department of Ophthalmology, Division of Ophthalmic Plastic and Reconstructive Surgery, John A. Moran Eye Center, University of Utah, Salt Lake City, UT, USA

3 Corneoplastic Unit, Queen Victoria Hospital, East Grinstead, UK description denotes a clinical phenomenon, in which, during any stage of blinking there is punctal apposition, preventing normal influx of tears through each of the puncta and consequently through the canalicular system. Patients may present with plerolacrima (symptomatic epiphora without tears running down the cheek), in mild cases or frank epiphora (symptomatic epiphora with excursion of tears over the eyelid margin onto the cheek) in severe cases. The management of epiphora in the presence of KP is not well reported in the literature.

Various authors have reported success with a variety of surgical procedures to restore anatomy and allow adequate function of the lacrimal drainage system. Ptosis surgery [1-3], horizontal lid laxity [4], surgical reduction of conjunctivochalasis [3, 4] and periorbital fat debulking [5] have been described in the context of KP.

It is intuitive to aim to restore the puncta to their premorbid anatomical positions using eyelid-tightening procedures, sometimes in conjunction with ptosis surgery. We describe our surgical experience of managing epiphora in the presence of KP. This interventional case series also aims 
Table 1 Clinical findings, management and outcomes in patients with 'kissing puncta'

\begin{tabular}{|c|c|c|c|c|c|}
\hline Case & 1 & 2 & 3 & 4 & 5 \\
\hline Age & 74 & 71 & 77 & 68 & 66 \\
\hline Gender & Female & Female & Male & Male & Male \\
\hline Laterality & Bilateral & Right & Bilateral & Bilateral & Bilateral \\
\hline Ptosis & + & + & + & - & + \\
\hline Lower eyelid laterality & + & - & ++ & ++ & ++ \\
\hline $\begin{array}{l}\text { Punctate corneal } \\
\text { epitheliopathy }\end{array}$ & ++ & ++ & + & - & - \\
\hline Type of epiphora & $\begin{array}{l}\text { Reflex, mainly during } \\
\text { activities and outdoors }\end{array}$ & $\begin{array}{l}\text { Medial spill over of } \\
\text { tears }\end{array}$ & $\begin{array}{l}\text { Medial spill over of } \\
\text { tears }\end{array}$ & $\begin{array}{l}\text { Lateral spill over } \\
\text { of tears }\end{array}$ & $\begin{array}{l}\text { Lateral spill over } \\
\text { of tears }\end{array}$ \\
\hline Intervention & Lateral tarsal strip & Endonasal DCR & Lateral tarsal strip & Lateral tarsal strip & Lateral tarsal strip \\
\hline Secondary intervention & - & - & Endonasal DCR & - & - \\
\hline $\begin{array}{l}\text { MUNK }^{\mathrm{a}} \text { score pre- } \\
\text { intervention }\end{array}$ & 4 & 2 & 4 & 4 & 1 \\
\hline $\begin{array}{l}\text { MUNK score post } \\
\text { intervention }\end{array}$ & 4 & 2 & 0 & 4 & 1 \\
\hline
\end{tabular}

${ }^{\mathrm{a} M U N K}$ score

to present a rationale to the management of this challenging cohort of patients.

\section{Methods}

A retrospective, multi-centre, audit of case notes of five patients (nine eyes) was conducted. Patients with symptomatic epiphora, to include those with nasolacrimal duct obstruction, in the context of punctal apposition were included. KP was defined as apposition of the punctum at primary gaze position or present in all phases of blink closure. Patients with pre-existing corneal pathology, punctal and nasolacrimal abnormalities, facial nerve dysfunction or thyroid abnormality were excluded.

\section{Results}

Five caucasian patients (three males and two females) with a mean age of 71 years (range: 66-77 years) were included. Patients were symptomatic with epiphora for at least 1 year, in the setting of KP. Four patients had bilateral disease and one patient had unilateral (right eye) epiphora (Table 1). None of the patients had any other significant associated symptoms related to their lacrimal function nor did they have a past history of lacrimal or adnexal disease.

Clinical examination confirmed punctal apposition in all five patients. Eight eyes $(80 \%)$ had clinically significant involutional, horizontal lower eyelid laxity in addition to punctal apposition. Medial upper eyelid ptosis with fat prolapse was present in four patients with a reduced MRD1 (distance from corneal light reflex to upper lid margin). Two patients had co-existent, partial nasolacrimal duct obstruction.

\section{Surgical intervention and outcomes}

Four patients with bilateral horizontal lower eyelid laxity underwent lower lid-tightening procedure with successful improvement in only one patient (two eyes) (Table 1). Of these, one symptomatic patient had bilateral partial nasolacrimal duct obstruction and underwent secondary surgery with endonasal dacrocystorhinostomy (DCR) resulting in improvement of symptoms.

One patient with unilateral epiphora, punctal apposition and partial nasolacrimal duct obstruction, endonasal DCR did not achieve improvement in symptoms despite a surgically patent lacrimal bypass system post-operatively (Table 1).

All patients showed clinical improvement in punctal apposition. However, epiphora symptoms had improved in one patients following endonasal DCR. This patient had an improvement in their MUNK score from 4 to 0. Epiphora continued in all the other patients. All patients had patent nasolacrimal system on syringing and in one patient, epiphora continued despite a patent lacrimal bypass system following endonasal DCR. Two of the four patients had a MUNK score of 1 with symptoms of epiphora when outdoors and two patients had a MUNK score of 4 .

\section{Discussion}

This paper reminds readers of the KP phenomenon with some new understanding of associated morbidity. Our 
Fig. 1 Colour photo showing the clinical signs associated with 'kissing puncta': (1) medial ptosis with fat prolapse, (2) horizontal lower eyelid laxity and (3) punctal apposition

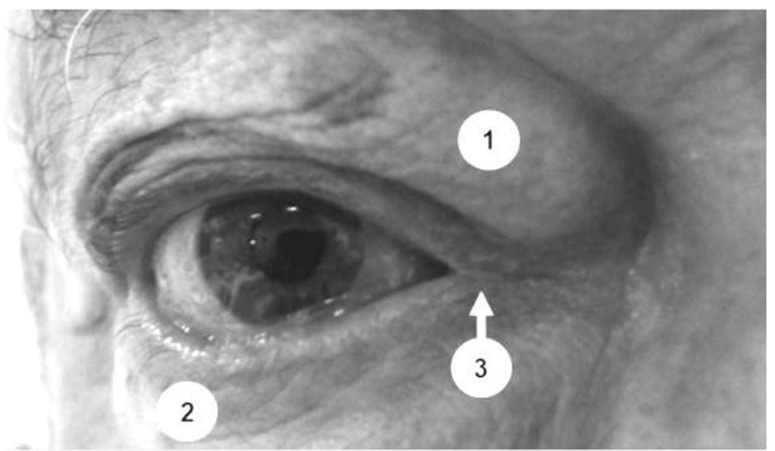

premise is that patients with $\mathrm{KP}$ have a mechanical obstruction of tear drainage. Our study reports guarded outcomes for resolution of epiphora following eyelid surgery in comparison to the few published papers in the literature. We also found a common triad of clinical signs associated with this cause of epiphora. We attribute KP to be strongly associated with (i) medial ptosis with fat prolapse, (ii) horizontal lower eyelid laxity and (iii) punctal apposition (Fig. 1).

Successful surgical correction to repair this clinical abnormality to pre-morbid state often necessitates more than one surgical procedure. Management of epiphora in patients with KP may prove challenging.

Glatt [3] reported KP to be associated with blepharoptosis. He first described the surgical management of KP in the literature in a patient with blepharoptosis. His patient was made symptom-free by surgical correction of blepharoptosis, which effectively elevated the superior punctum from the inferior punctum to restore the puncta to their normal anatomy, thereby helping tear drainage. The prevalence of punctal apposition is unknown. Similarly, it is unknown if apposition of the puncta is more common in overweight patients due to multiple factors (fat prolapse, dermatochalasis and loss of elasticity). It is likely that this anatomical abnormality would be present in patients who do not report epiphora. It is possible that punctal apposition simply represents another feature of involutional age-related change and may have a variable causal relationship with epiphora. It should not be assumed that epiphora in the presence of KP is primarily due to simple mechanical punctal obstruction to the tear flow.

Bartley [1] first described the concept of punctal apposition as a secondary mechanical cause of acquired lacrimal drainage obstruction. Surgical correction of these clinical abnormalities have been reported in the literature with success. These surgical interventions included ptosis correction [2, 3], Muller's muscle resection [4], lid tightening [5] and periorbital fat debulking [7]. Conjunctivochalasis in some studies was found be an additional cofactor, impeding normal tear flow. Putterman and Francis [4, 5] carried out conjunctival resection as part of their surgical intervention. These studies have reported successful amelioration of epiphora in their group of patients.

The clinical triad of medial ptosis with fat prolapse, horizontal lower eyelid laxity and punctal apposition should make the clinician aware of the likelihood of KP as a possible contributory cause of epiphora. However, based upon the findings of this study, patients should be counselled that restoration of normal punctal position with reversal of KP may not resolve symptoms. We attribute the lack of improvement in symptoms to multifactorial causes of epiphora. Two of our patients (four eyes) had ocular surface disease, which could have contributed to their epiphora, two had partial nasolacrimal duct obstruction and lacrimal bypass surgery resolved symptoms in one patient and continued in the other despite a clinically patent nasolacrimal system on syringing.

Our findings are in contrast to previous reported findings [2-7] and highlights the complexities involved in managing this cohort of patients. The striking difference in our surgical outcomes may represent a small cohort within a spectrum of patients with KP.

Successful combined surgery in the setting of epiphora and KP by Putterman and Francis [4, 5] sheds light on the importance of considering multifactorial causes of epiphora. KP should not be thought of as a sole cause of obstruction to tear flow. Other forms of lacrimal tear drainage impediment must be considered as well as causes of non-drainage obstruction, such as reflex tearing due to ocular surface disease. A useful way of evaluating and classifying epiphora is by the use of the mnemonic BLICK (blink dynamics, lid malposition, imbrication, conjunctivochalasis and KP) as described by Tse et al. [8].

In conclusion, this paper describes $\mathrm{KP}$ as an important, under-reported, clinical abnormality leading to mechanical obstruction of lacrimal drainage and epiphora. We describe a triad of clinical signs associated with KP. Resolution of epiphora in these patients may be challenging and appropriate counselling of a guarded prognosis is suggested. 


\section{Summary}

\section{What was known before}

- There is limited knowledge of the challenges of managing epiphora caused by kissing puncta (punctal apposition).

\section{What this study adds}

- This study describes for the first time the clinical triad associated with kissing puncta.

- This study illustrates the challenges of surgical correction to alleviate epiphora.

- This study makes recommendations based on second point above.

\section{Compliance with ethical standards}

Conflict of interest The authors declare that they have no conflict of interest.

\section{References}

1. Bartley GB. Acquired lacrimal drainage obstruction: an etiologic classification system, case reports, and a review of the literature. Part 3. Ophthalmic Plat Reconstr Surg. 1993;1: $11-26$.

2. Hurwitz JJ. Examination of the eyelids. The lacrimal system. Philadelphia: Lippincott-Raven; eds. Jeffrey Jay Hurwitz, 1996. p. 48.

3. Glatt HJ. Epiphora caused by blepharoptosis (letter). Am J Ophthalmol. 1991;111:649-50.

4. Putterman A. Epiphora caused by blepharotosis. Am J Ophthalmol. 1991;111:649-50. 49

5. Francis IC, Wan MK. The punctal apposition syndrome: a new surgical approach. Br J Ophthalmol. 2002;86:1256-8.

6. Munk PL, Lin DT, Morris DC. Epiphora: treatment by means of dacryocystoplasty with balloon dilatation of the nasolacrimal drainage apparatus. Radiology. 1990;177:687-90.

7. Cheema MM, Meyer DR. Epiphora secondary to punctal apposition in the setting of Graves'orbitopathy. Ophthalmic Plast Reconstr Surg. 1995;11:122-4.

8. Tse D, Erickson B, Tse B. The BLICK mneumonic for clinical anatomical assessment of pa-tientswith epiphora. Ophthalmic Plast Reconstr Surg. 2014;30:450-8. 